\title{
NOTAS SÔBRE A PESCA DA CAVALA E DA SERRA NO CEARÁ - DADOS DE $1969^{(1)}$
}

\author{
Raimundo Saraiva da Costa - Melquíades Pinto Paiva
}

\author{
Laboratório de Ciências do Mar \\ Universidade Federal do Ceará \\ Fortaleza - Ceará - Brasil
}

Com o presente trabalho, o Laboratório de Ciências do Mar da Universidade Federal cio Ceará dá prosseguimento à série de estudos sôbre a pesca da cavala, Scomberomorus cavalla (Cuvier), e da serra, Scomberomorus maculatus (Mitchill), efetuada em águas costeiras do Estado do Ceará, Brasil.

Ressaltamos que durante o ano de 1969 a pesca dêsses peixes foi conduzida dentro do padrão tradicional, com embarcações, artes e métodos primitivos.

\section{MATERIAL}

O material que serve de fundamento a êste trabalho foi capturado em frente ao município de Fortaleza, tendo sido desembarcado na praia de Mucuripe. Corresponde a amostras de 236 dias de pescarias, abrangendo os meses de janeiro a dezembro de 1969 . As pescarias foram realizadas por jangadas e alguns botes motorizados, operando com linhas de corso. Foram amostradas 5.181 cavalas e 1.886 serras (tabelas I e II). Também, controlamos 2.278 pescarias de jangadas, realizadas dentro do padrão referido, na mesma área e durante os meses de janeiro a dezembro de 1969 (tabela $\mathrm{V}$ ).

\section{MÉTODO}

Em cada amostra procuramos incluir o máximo possível de indivíduos de ambas as espécies, desembarcados na praia de Mucuripe, e registrados à tarde, após a chegada das embarcações que volviam da faina diária de pesca.

(1) - Trabalho realizado em decorrència de convênios celebrados com a Superintendência do Desenvolvimento do Nordeste (SUDENE) e a Superintendência do Desenvolvimento da Pesca (SUDEPE).
Durante a obtenção das amostras, para cada indivíduo, anotamos apenas a espécie e o comprimento zoológico. Por serem espécjmens de alto valor comercial e de demanda imediata, o conhecimento dos sexos estavam acima das nossas possibilidades, uma vez que eram vendidos antes mesmo da evisceração.

Consideramos como comprimento zoolócico (fork length) a distância entre o extremo anterior da cabeça e o extremo posterior dos raios medianos da caudal, estando o animal estendido lateralmente sôbre uma superfície plana.

Os comprimentos zoológicos dos indivíciuos amostrados, de ambas as espécies, foram agrupados, segundo as variações apresentadas, ros respectivos grupos de idades. A aplicação dêste procedimento baseia-se nos trabalhos de Nomura \& Rodrigues (1967) e Nomura (1967), para a cavala e serra, respectivamente.

O contrôle das pescarias foi efetuado diàriamente, na praia de Mucuripe, logo após a chegada das embarcações selecionadas.

De cada pescaria realizada anotamos, principalmente, o número de indivíduos capturados de cavala e de serra, o número de anzóis empregados e o tipo de isca usado, cujos dados foram agrupados por meses.

Os índices de densidade relativa, determinados para cada espécie, foram calculados a partir dos totais de indivíduos mensalmente capturados por centena de anzóis/dia empregados.

\section{DISCUSSÃO E CONCLUSÕES}

Segundo Albuquerque \& Bezerra (1968) a amostragem mínima mensal para a cavala ६ a serra, no Estado do Ceará, deve ser de 400 e 300 indivíduos, respectivamente. Sendo assim, no tocante à cavala, tal exigência foi atendida nos meses de fevereiro, maio, junho 


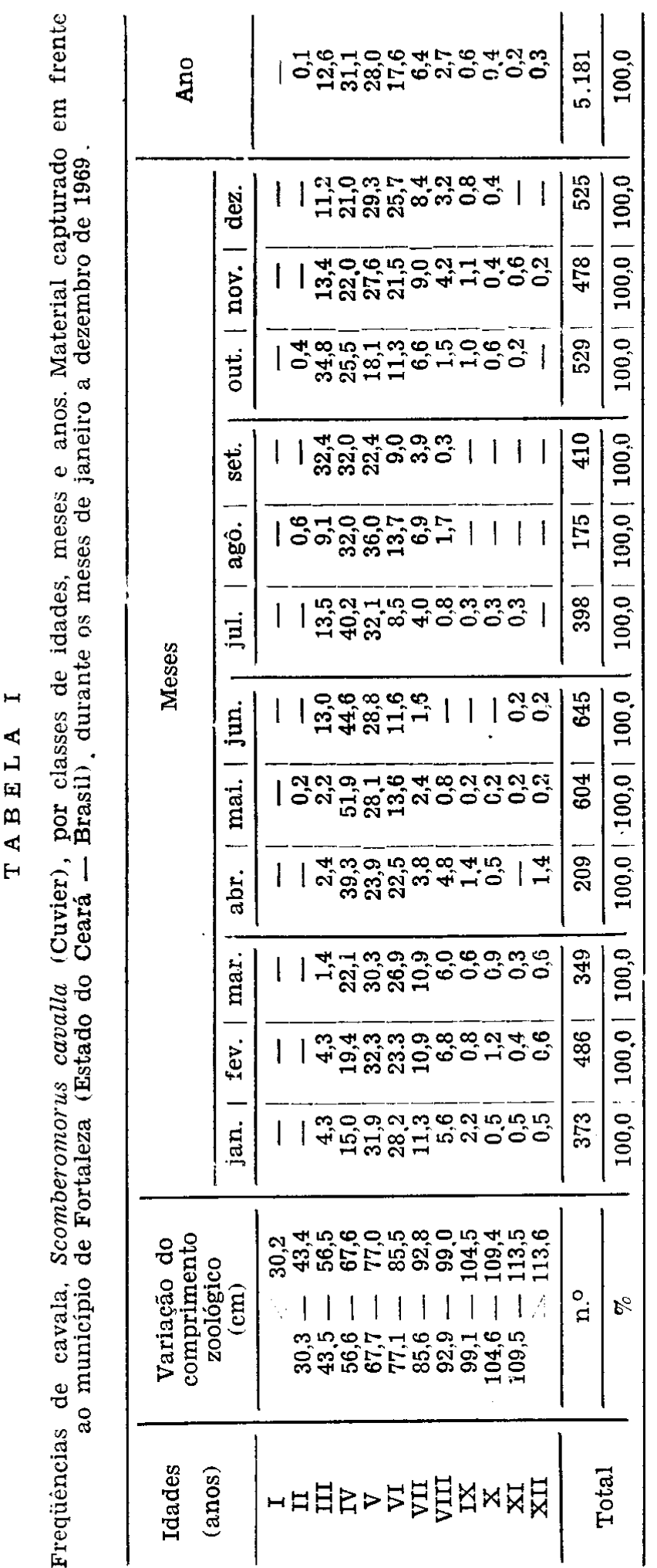

e no periodo de setembro a dezembro; e para a serra somente no mês de novembro (tabelas I e II) .

Para a cavala, os grupos de idades atingidos pela pesca, no conjunto geral das amostras, variaram entre os correspondentes a II e XII anos, concentrando-se entre os de III e VII anos, com máximo entre os IV e V anos (tabela I, figura 1) . Para a serra, êstes grupos

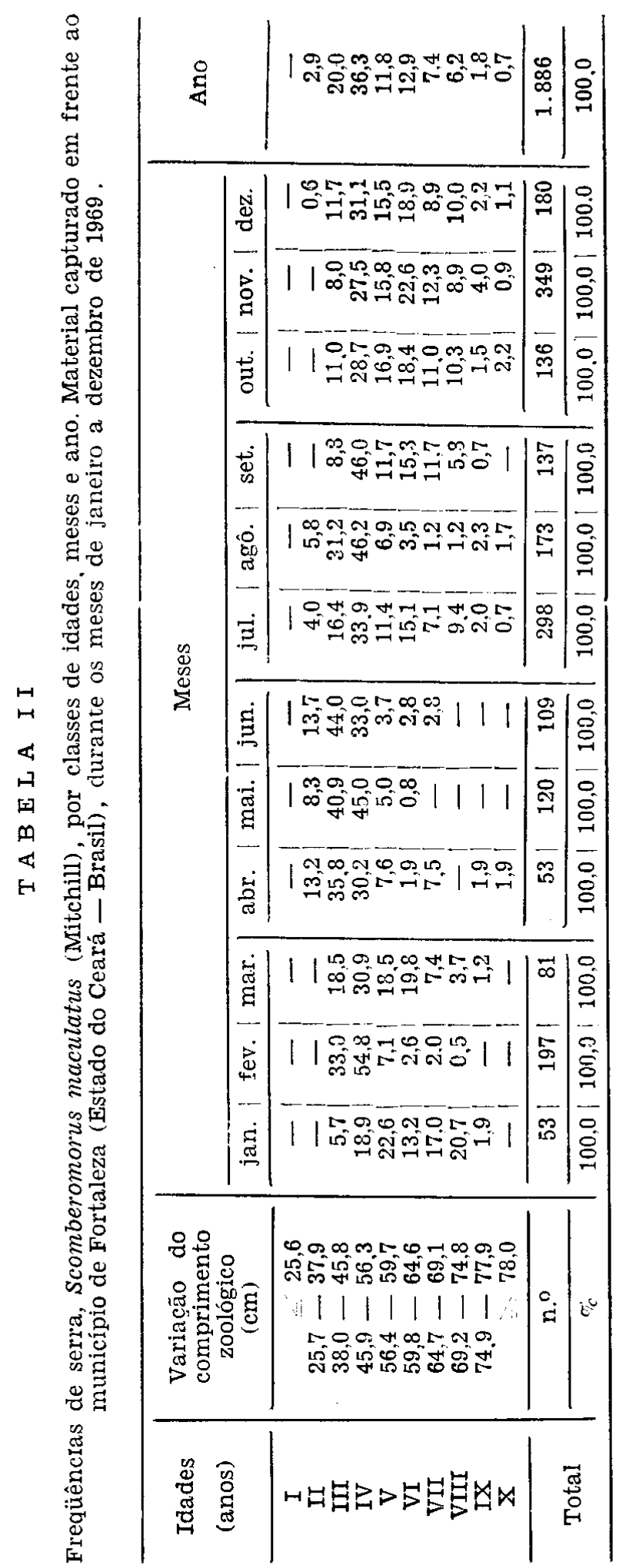

variaram entre os correspondentes a II e $\mathrm{X}$ anos, concentrando-se entre os de III e VIII anos, com máximo entre os de III e IV anos (tabela II , figura 1) .

Nas pescarias da serra ao sul da Flórida (U. S.A.), os cinco primeiros grupos de idades são os que compõem os desembarques (Klima: נ.959) . 
T A B E I A I I I

Dados mensais referentes aos comprimentos zoológicos (cm) de cavala, Scomberomorus cavalla (Cuvier). Material capturado em frente ao município de Fortaleza (Estado do Ceará - Brasil), durante os meses de janeiro a dezembro de 1969 .

\begin{tabular}{|c|c|c|c|c|c|c|}
\hline \multirow[b]{2}{*}{ Meses } & \multirow{2}{*}{$\begin{array}{l}\text { Número de } \\
\text { indivíduos } \\
\text { (n) }\end{array}$} & \multicolumn{5}{|c|}{ Comprimento zoológico $(\mathrm{cm})$} \\
\hline & & $\begin{array}{l}\text { valor } \\
\text { maximo }\end{array}$ & $\begin{array}{l}\text { valor } \\
\text { mínimo }\end{array}$ & $\begin{array}{l}\text { média } \\
\text { aritmética } \\
(\bar{x})\end{array}$ & $\begin{array}{l}\text { desvio } \\
\text { padrão } \\
\text { ( S ) }\end{array}$ & $\begin{array}{l}\text { coeficiente } \\
\text { de variação } \\
\text { (C.V.) }\end{array}$ \\
\hline $\begin{array}{l}\text { janeiro } \\
\text { fevereiro } \\
\text { março } \\
\text { abril } \\
\text { maio } \\
\text { junho } \\
\text { julho } \\
\text { agôsto } \\
\text { setembro } \\
\text { outubro } \\
\text { novembro } \\
\text { dezembro }\end{array}$ & $\begin{array}{l}373 \\
486 \\
349 \\
209 \\
604 \\
645 \\
398 \\
175 \\
410 \\
529 \\
478 \\
525\end{array}$ & $\begin{array}{r}135,3 \\
127,8 \\
120,5 \\
126,2 \\
112,6 \\
146,3 \\
112,2 \\
94,8 \\
91,8 \\
112,3 \\
122,0 \\
107,7\end{array}$ & $\begin{array}{l}48,6 \\
44,7 \\
53,1 \\
51,3 \\
50,3 \\
50,2 \\
48,4 \\
38,2 \\
45,8 \\
38,4 \\
46,9 \\
47,2\end{array}$ & $\begin{array}{l}77,1 \\
76,1 \\
76,4 \\
73,0 \\
68,7 \\
66,9 \\
67,2 \\
69.2 \\
63,5 \\
65,1 \\
72,9 \\
72,9\end{array}$ & $\begin{array}{r}11,89 \\
12,43 \\
11,23 \\
12,50 \\
9,10 \\
9,75 \\
10,00 \\
9,40 \\
11,10 \\
14,00 \\
13,10 \\
11,75\end{array}$ & $\begin{array}{l}15,42 \\
16,33 \\
14,69 \\
17,12 \\
13,24 \\
14,50 \\
14,88 \\
13,58 \\
17,48 \\
21,51 \\
17,97 \\
16,12\end{array}$ \\
\hline Total & 5.181 & 146,3 & 38,2 & 70,4 & 12,30 & 17,47 \\
\hline
\end{tabular}

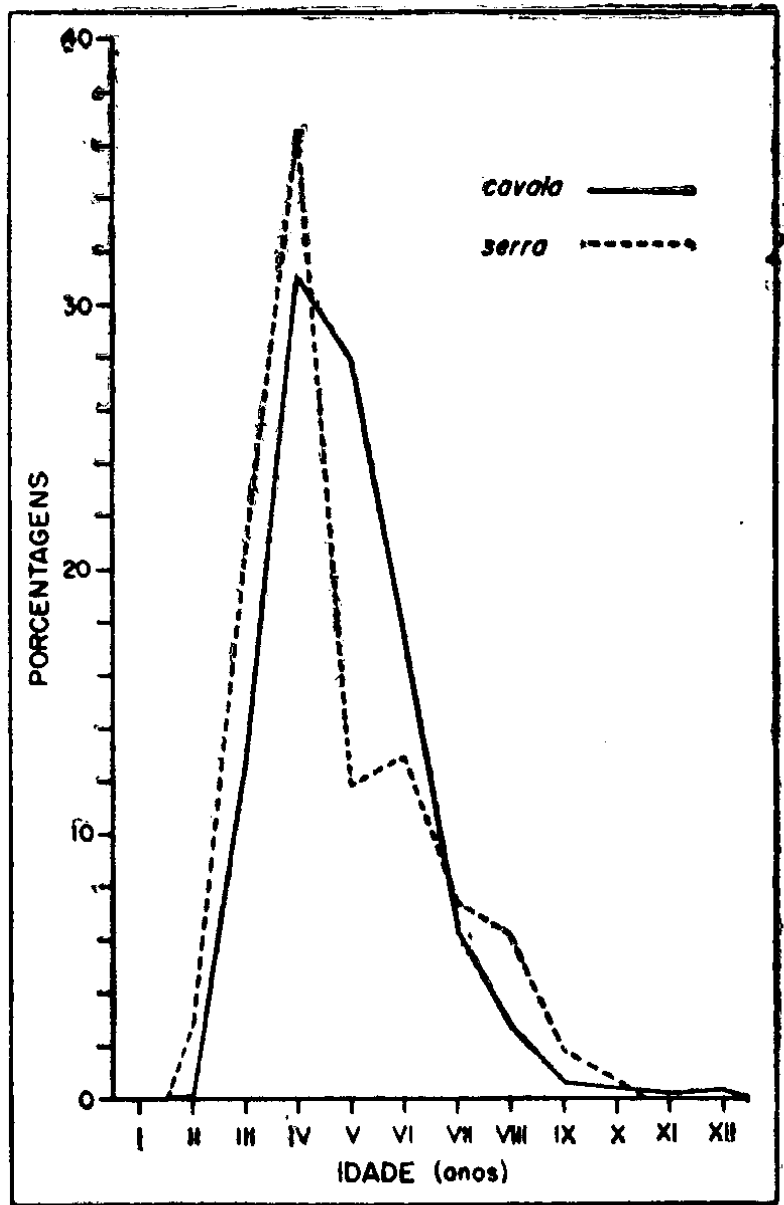

Figura 1 - Freqüências relativas de indivíduos das espécies Scomberomorus cavalla (Cuvier) e Scomberomorus maculatus (Mitchill), por grupos de idades, no conjunto geral das amostras. Material capturado em frente ao município de Fortaleza (Estado do Ceará - Brasil), no período de janeiro a dezembro de 1969 .
Apesar do número de indivíduos amostrados, de cada espécie em estudo, ter variado muito entre os meses, as médias aritméticas dos comprimentos zoológicos, bem como seus desvios padrões e coeficientes de variação são muito próximos (tabelas III e IV , figura 2) . Para a cavala, a maior destas médias foi a do mês de janeiro, quando atingiu o valor de $77,1 \mathrm{~cm}$, e a menor correspondeu ao mês de setembro, expressa pelo valor de $63,5 \mathrm{~cm} . \mathrm{Em}$ relação à serra, a maior destas médias foi a cio mês de janeiro, quando atingiu o valor de $61,1 \mathrm{~cm}$, e a menor correspondeu ao mês de junho, expressa pelo valor de $45,2 \mathrm{~cm}$.

Os totais de anzóis/dia empregados nas pescarias controladas, em geral, variaram em relação direta com as freqüências mensais das pescarias; as médias mensais de anzóis utilizados por pescaria se apresentaram mais ou menos constantes; a média anual de anzóis por pescaria foi de 8,0 anzóis (tabela V). Os tipos de anzóis mais utilizados, nas pescarias

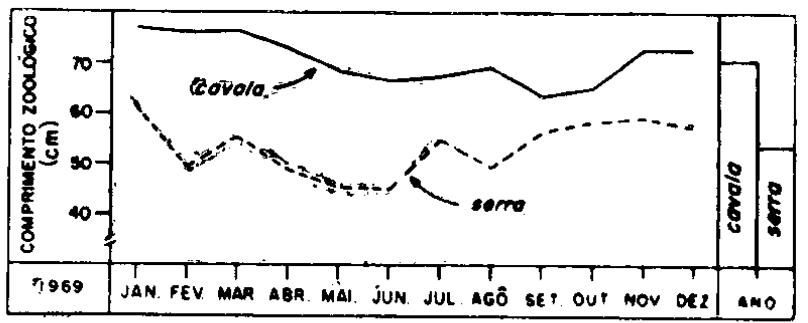

Figura 2 - Distribuição das médias aritméticas de comprimentos zoológicos de indivíduos das espécies Scomberomorus cavalla (Cuvier) e Scomberomorus maculatus (Mitchill), correspondentes aos diversos conjuntos mensais de amostras. Material capturado em trente ao município de Fortaleza (Estado do Ceará - Brasil), no período de janeiro a dezembro de 1969 . 


\section{T A B E L A I V}

Dados mensais referentes aos comprimentos zoológicos (cm) de serra, Scomberomorus maculatus (Mitchill). Material capturado em frente ao município de Fortaleza (Estado do Ceará — Brasil), durante os meses de janeiro a dezambro de 1969 .

\begin{tabular}{|c|c|c|c|c|c|c|}
\hline \multirow[b]{2}{*}{ Meses } & \multirow[b]{2}{*}{$\begin{array}{l}\text { Número de } \\
\text { indivíduos } \\
\text { (n) }\end{array}$} & \multicolumn{5}{|c|}{ Comprimento zoológico $(\mathrm{cm})$} \\
\hline & & $\begin{array}{l}\text { valor } \\
\text { máximo }\end{array}$ & $\begin{array}{l}\text { valor } \\
\text { minimo }\end{array}$ & $\begin{array}{c}\text { média } \\
\text { aritmética } \\
(\overline{\mathrm{x}})\end{array}$ & $\begin{array}{l}\text { desvio } \\
\text { padrão } \\
\text { ( s ) }\end{array}$ & $\begin{array}{l}\text { coeficiente } \\
\text { de variação } \\
\text { (C.V.) }\end{array}$ \\
\hline $\begin{array}{l}\text { janeiro } \\
\text { fevereiro } \\
\text { março } \\
\text { abril } \\
\text { maio } \\
\text { junho } \\
\text { julho } \\
\text { agôsto } \\
\text { setembro } \\
\text { outubro } \\
\text { novembro } \\
\text { dezembro }\end{array}$ & $\begin{array}{r}53 \\
197 \\
81 \\
53 \\
120 \\
109 \\
298 \\
173 \\
137 \\
136 \\
349 \\
180\end{array}$ & $\begin{array}{l}76,1 \\
74,4 \\
76,3 \\
80,5 \\
62,5 \\
65,3 \\
79,4 \\
85,8 \\
77,1 \\
82,3 \\
85,6 \\
80,7\end{array}$ & $\begin{array}{l}39,6 \\
40,9 \\
39,9 \\
30,2 \\
30,6 \\
34,8 \\
34,7 \\
35,7 \\
38,8 \\
41,3 \\
39,9 \\
37,6\end{array}$ & $\begin{array}{l}61,1 \\
49,5 \\
55,2 \\
48,9 \\
45,6 \\
45,2 \\
54,9 \\
49,3 \\
56,3 \\
58,5 \\
59,3 \\
57,7\end{array}$ & $\begin{array}{r}8,87 \\
5,84 \\
8,63 \\
10,55 \\
5,25 \\
6,90 \\
10,60 \\
8,80 \\
8,35 \\
9,25 \\
8,35 \\
9,50\end{array}$ & $\begin{array}{l}14.52 \\
11,80 \\
15,63 \\
21,57 \\
11,40 \\
15,26 \\
19,30 \\
17,85 \\
14,85 \\
15,81 \\
1.08 \\
16,46\end{array}$ \\
\hline Total & 1.886 & 85,8 & 30,2 & 53,9 & 9,95 & 18,46 \\
\hline
\end{tabular}

\section{T A B E L A V}

Dados de pescarias controladas de cavala, Scomberomorus cavalla (Cuvier), e de serra, Scomberomorus maculatus (Mitchill), realizadas por embarcações que operaram em frente ao município de Fortaleza (Estado do Ceará - Brasil), durante os meses de janeiro a dezembro de 1969 .

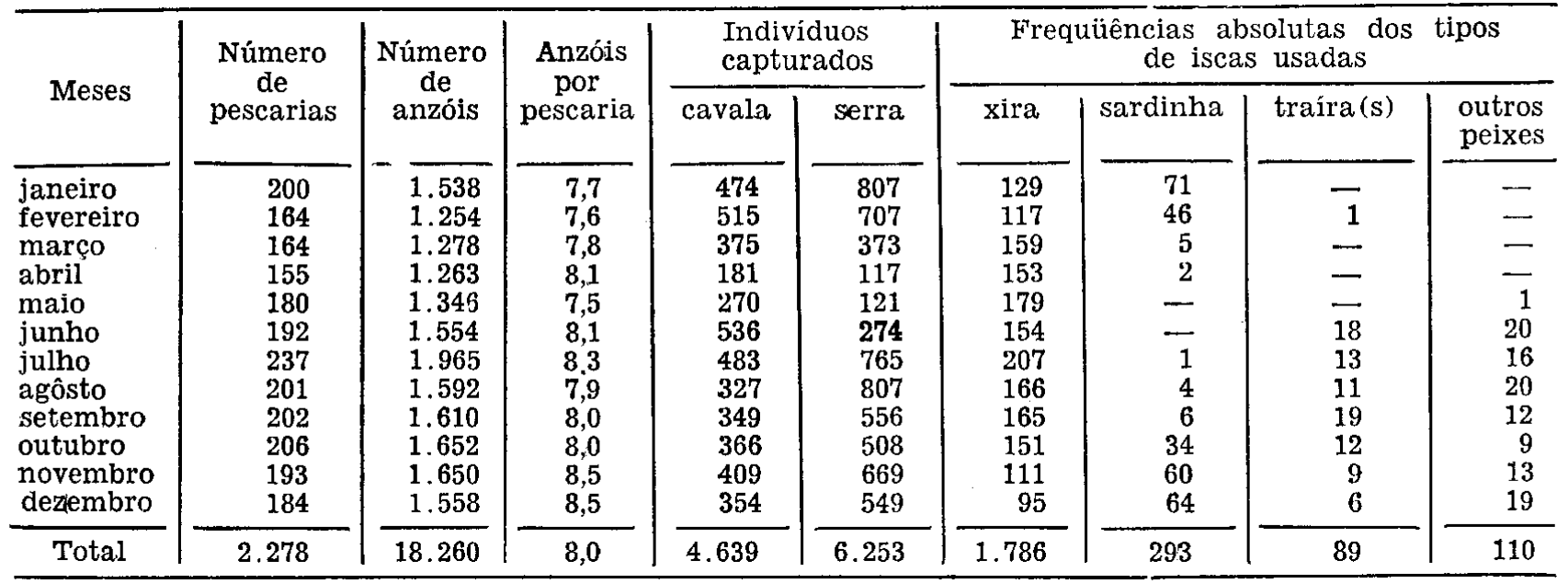

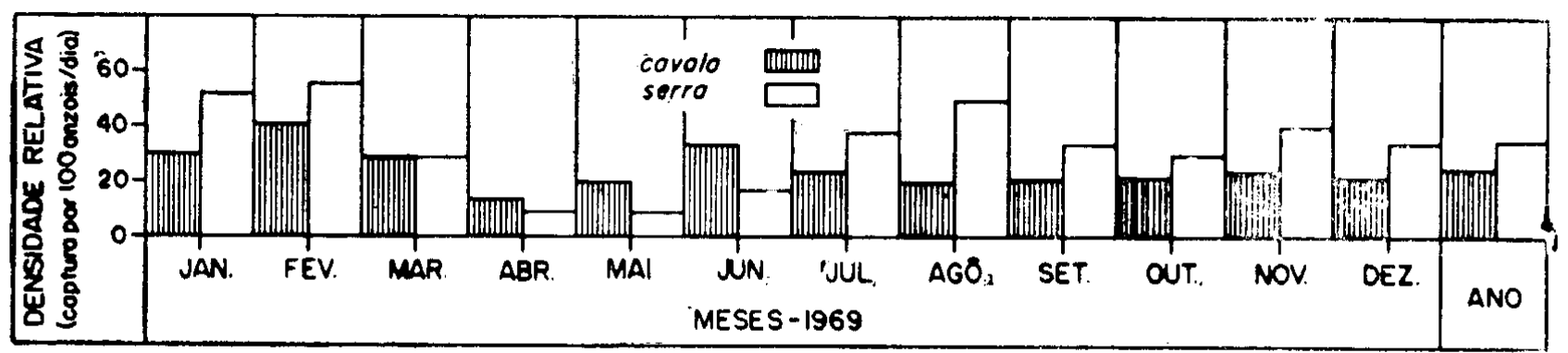

Figura 3 - Distribuição dos indices de densidade relativa (captura por 100 anzóis/dia) das espécies Scomberomorus cavalla (Cuvier) e Scomberomorus maculatus (Mitchill). Material capturado em frente as município de Fortaleza (Estado do Ceará — Brasil), no período de janeiro a dezembro de 1969 . 
T A B E L A V I

Indices mensais de captura por pescaria e de densidade relativa (captura por 100 anzóis/dia) de cavala. Scomberomorus cavalla (Cuvier), e de serra, Scomberomorus maculatus (Mitchill), na área de Fortaleza (Estado do Ceará — Brasil), durante os meses de janeiro a dezembro de 1969 .

\begin{tabular}{|c|c|c|c|c|}
\hline \multirow[t]{2}{*}{ Meses } & \multicolumn{2}{|c|}{$\frac{\text { Número de indivíduos capturados }}{\text { número de pescarias }}$} & \multicolumn{2}{|c|}{$\begin{array}{l}\text { Densidade relativa } * \\
\text { (captura por } 100 \text { anzóis/dia) }\end{array}$} \\
\hline & cavala & serra & cavala & serra \\
\hline $\begin{array}{l}\text { janeiro } \\
\text { fevereiro } \\
\text { março } \\
\text { abril } \\
\text { maio } \\
\text { junho } \\
\text { julho } \\
\text { agôsto } \\
\text { setembro } \\
\text { outubro } \\
\text { novembro } \\
\text { dezembro }\end{array}$ & $\begin{array}{l}2,4 \\
3,1 \\
2,3 \\
1,2 \\
1,5 \\
2,8 \\
2,0 \\
1,6 \\
1,7 \\
1,8 \\
2,1 \\
1,9\end{array}$ & $\begin{array}{l}4.0 \\
4.3 \\
2,3 \\
0,8 \\
0,7 \\
1,4 \\
3,2 \\
4,0 \\
2,3 \\
2,5 \\
3.5 \\
3,0 \\
\end{array}$ & $\begin{array}{l}30,8 \\
41,1 \\
29,3 \\
14,3 \\
20,0 \\
34,5 \\
24,6 \\
20,5 \\
21,7 \\
22,2 \\
24,8 \\
22,7\end{array}$ & $\begin{array}{r}52,5 \\
56,4 \\
29,2 \\
9,3 \\
9,0 \\
17,6 \\
38,9 \\
50,7 \\
34,5 \\
30,8 \\
40,5 \\
35,2\end{array}$ \\
\hline Total & 2,0 & 2,7 & 25,4 & 34,2 \\
\hline
\end{tabular}

" Deixaram de ser consideradas as outras espécies capturadas.

controlacias, foram os de números 4 e 5 para a cavala, e os de números 6 e 7 para a serra.

As iscas usadas nas pescarias controladas foram as seguintes: xira - Haemulon aurolineatum Cuvier, sardinha - Opisthonema oglinum (Le Sueur) e traíra(s) - Trachinocephalus myops (Forster) e/ou Synodus intermedius (Agassiz), em ordem decrescente de importância, sendo também empregados outros pequenos peixes marinhos, em escala muito pequena, podendo mesmo não serem tomados em consideração. As freqüências mensais dos tipos de isca usados nas pescarias, mostram que, em todos os meses, a xira predominou sôbre os demais tipos (tabela $\mathrm{V}$ ) .

Nas pescarias controladas, somente foram consideradas as capturas da cavala e da serra (tabela V) .

Os valôres encontrados para a relação de indivíduos capturados/número de pescarias e para a densidade relativa - captura por 100 anzóis/dia (tabela VI, figura 3), evidenciam que a captura por pescaria depende da densidade relativa.

Os mais altos índices de densidade relativa da cavala corresponderam aos meses fevereiro e junho; para a serra foram os d: meses de janeiro, fevereiro, agôsto e novembro

\section{SUMMARY}

With this paper the Marine Sciences Laboratory of the Federal University of Ceará carries on a serial study on the fishery biology of the king mackerel, Scomberomorus cavalla (Cuvier), and Spanish mackerel, Scomberomorus maculatus (Mitchill), in the State of Ceará, Brazil. 'i'he material was caught off Fortaleza, and landed at Mucuripe Beach. It corresponds to samples of 236 days of fishing carried out ciuring the period from January to December, 1969 , made by rafts and some motor boats. We sampled 5,181 king mackerels and 1,886 Spanish mackerels. We have also controlled 2,278 rafts fishings, in the same area and throughout the months from January to December, 1969 .

For the king mackerel the age groups caught ranged from II to XII years, being observed concentration between III and VII years, and maximum between IV and $V$ years.

For the Spanish mackerel, the age groups caught ranged from II to $X$ years, being observed concentration between III and VIII years, and maximum between III and IV years.

In the several monthly set of samples, the arithmetic means of fork lengths corresponding to each one of the species, as well as its standard deviations and variation coefficients are very close. For the king mackerel the greatest among those arithmetic means corresponded to the month of January, when reached value of $77.1 \mathrm{~cm}$, and the smallest one was reported in the month of September, expressed through the value of $63.5 \mathrm{~cm}$. For the Spanish mackerel, the greatest among those arithmetic means corresponded to the month of January, when reached the value of $61.1 \mathrm{~cm}$, an the smallest cne was reported in the month of June, expressed through the value of $45.2 \mathrm{~cm}$.

The total number of hooks monthly used

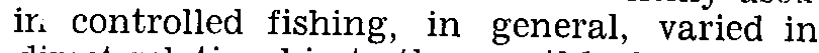
cirect relationship to the monthly frequencies of fishings. The monthly means of the number 
of hooks used in each fishing were more cr less constant. The year mean of hooks in each fishing was 8.0 hooks.

The types of bait used in controlled fishings were the tomtate - Haemulon aurolineatum Cuvier, the Atlantic thread-herring - Opisthonema oglinum (Le Sueur), and snakefish - Trachinocephalus myops (Forster) and/or sand diver - Synodus intermedius (Agassiz), in decreasing order of importance, being used other marine small fish, in less number.

In the controlled fishings were considered only the king mackerel and Spanish mackerel catches, reaching the totals of $4,639 \mathrm{king}$ mackerels and 6,253 Spanish mackerels.

We have studied the relations number of caught individuals/number of fishings and number of individuals caught by 100 hooks/ /day (relative density). The catches by fishing depended of the relative - density indexes. The highest numbers of relative density for the king mackerel corresponded to the months of February and June; for the Spanish mackerel, to the months of October, November and December.

\section{BIBLIOGRAFIA CONSULTADA}

Albuquerque, J. J. L. \& Bezerra, R. C. F. 1968 - Sôbre a amostragem da cavala e da serra no Ceará (Pisces) . Rev. Brasil. Biol., Rio de Janeiro, 28 (2): 141-145. 2 figs.
Costa, R. S. \& Paiva, M. P. - 1963 - Notas sôbre a pesca da. cavala e da serra no Ceará Dados de 1962. Arq. Est. Biol. Mar. Univ. Ceará, Fortaleza, 3 (1) : 17-26, 4 figs.

Costa, R. S. \& Paiva, M. P. - 1964 - Notas sôbre a pesca da cavala e da serra no Ceará - Dados de 1963. Arq. Est. Biol. Mar. Univ. Ceará, Fortaleza 4 (2): $71-81,5$ figs.

Costa, R. S. \& Paiva, M. P. - 1965 - Notas sôbre a pesca da cavala e da serra no Ceará - Dados de 1964. Arq. Est. Biol. Mar. Univ. Ceará, Fortaleza, 5 (2): 93-101, 5 figs.

Costa, R. S. \& Paiva, M. P. - 1966 - Notas sôbre a pesca da cavala e da serra no Ceará Dados de 1965. Arq. Est. Biol. Mar. Univ. Fed. Ceará, Fortaleza, 6 (2) : 195-204 . 4 figs.

Costa, R. S. \& Paiva, M. P. - 1967 - Notas sôbre a pesca da cavala e da serra no Ceará - Dados de 1966 . Arq. Est. Biol. Mar. Univ. Fed. Ceará, Fortaleza, 7 (2) : 181-190, 4 figs.

Costa. R. S. \& Paiva, M. P. - 1968 - Notas sôbre a pesca da cavala e da serra no Ceará - Dados de 1967. Arq. Est. Biol. Mar. Univ. Fed. Cearí, Fortaleza, 8 (2): 125-131, 3 figs.

Costa, R. S. \& Paiva, M. P. - 1969 - Notas sôbre a pesca da cavala e da serra no Ceará - Da.dos de 1968. Arq. Ciên. Mar, Fortaleza, 9 (1): 89-95, 3 figs.

Klima, E. F. - 1959 - Aspects of the biology and the fishery for Spanish mackerel, Scomberomorus maculatus (Mitchill) of Southern Florida. State of Florida Board of Conservation Technical Series, Coral Gables, (27) : 1-39, 17 figs.

Nomura, H. - 1967 - Dados biológicos sôbre a serra, Scomberomorus maculatus (Mitchill), das águas cearenses. Arq. Est. Biol. Mar. Univ. Fed. Ceará, Fortaleza, 7 (1) : 29-39 4 figs.

Nomura, H. \& Rodrigues, M. S. S. - $1967-$ Biological notes on king mackerel, Scomberomorus cavalla (Cuvier), from northeastern Brazil. Arq. Est. Biol. Mar. Univ. Fed. Ceará, Fortaleza, 7 (1) : $79-85,4$ figs. 